\title{
Redes económicas solidarias: el caso de Brasil
}

\author{
Ivette Tatiana Castilla-Carrascal ${ }^{\star}$
}

* Doctoranda en Ciencias Sociales y Estudios Comparados de las Américas CEPPAC, Universidad de Brasilia. Profesora, Maestría Virtual en Economía Solidaria y Desarrollo Territorial, Universidad Cooperativa de Colombia. Investigadora del Centro de Estudios Comparados de las Américas ceppac, Universidad de Brasilia, Brasil.

Correo electrónico:

tatianacastillac@yahoo.com

Recibido: 25 de agosto del 2014

Aprobado: 7 de noviembre del 2014

Cómo citar este artículo: Castilla-Carrascal, I. T. (2014). Redes económicas solidarias: el caso de Brasil. Cooperativismo \&

Desarrollo, 22(105), 55-65. doi: http://dx.doi. org/10.16925/co.v22i105.1035

\section{Resumen}

Este artículo tiene como objetivo presentar algunas reflexiones (resultado de la disertación de la Maestría en Sociología) sobre diversas contribuciones de las redes de economía solidaria en la construcción de un mercado solidario en el Brasil. En la primera parte del artículo se realiza una discusión sobre las diferentes características de las redes en las teorías sociales más generales, así como de las redes en el campo de la economía solidaria. En la segunda parte del artículo se caracterizan brevemente las Redes Bodega, Acs Amazonia, Rede Ecovida, Central do Cerrado y Justa Trama, mostrando sus prácticas de comercio justo y solidario, su historia y los actores que se relacionan. Finalmente, en las consideraciones se presentan algunos de los desafíos enfrentados por las redes, así como impactos que los actores parte de las redes experimentan al interior de estas.

Palabras clave: comercio justo, economía solidaria, mercado solidario, redes de economía solidaria.

\section{Solidarity Economy Networks: the Case of Brazil}

\section{Abstract}

This article presents reflections (resulting from a Master's dissertation in sociology) about diverse contributions by the solidarity economy networks in the construction of a solidarity market in Brazil. The first part of the article is a discussion about the different characteristics of the networks in terms of more general social theories, such as networks in the field of the solidarity economy. The second part briefly characterizes the Redes Bodega, Acs Amazonia, Rede Ecovida, Central do Cerrado and Justa Trama networks, showing their fair trade and solidarity trade practices, history and the actors involved. Finally, the considerations present certain challenges faced by the networks, along with impacts that the actors in the networks experience within them.

Keywords: fair trade, solidarity economy, solidarity market, solidarity economy networks.

\section{Redes econômicas solidárias: o caso do Brasil}

\section{Resumo}

Este artigo tem como objetivo apresentar algumas reflexões (resultado da dissertação de mestrado em Sociologia) sobre diversas contribuições das redes de economia solidária na construção de um mercado solidário no Brasil. Na primeira parte do artigo, realiza-se uma discussão sobre as diferentes características das redes nas teorias sociais mais gerais, bem como das redes no campo da economia solidária. $\mathrm{Na}$ segunda parte, caracterizam-se brevemente as Redes Bodega, ACs Amazônia, Rede Ecovida, Central do Cerrado e Justa Trama, e mostram-se suas práticas de comércio justo e solidário, sua história e os atores que se relacionam. Finalmente, nas considerações, apresentam-se alguns dos desafios enfrentados pelas redes e os impactos que os seus atores experimentam no interior destas.

Palavras-chave: comércio justo, economia solidária, mercado solidário, redes de economia solidária. 


\section{Introducción}

Este artículo se propone presentar algunas contribuciones teóricas para la comprensión de las redes económicas solidarias, así como traer algunas experiencias que fueron analizadas en la investigación de maestría ${ }^{1}$. En la primera parte del artículo, se abarca el tema de las redes de manera más general, haciendo énfasis en sus características, principios e incentivos para la organización en red. En la segunda parte, se presentan cinco experiencias de redes de comercialización solidaria en Brasil, las cuales constituyeron el objeto de la investigación. Se presenta su historia, su organización y su funcionamiento. Por último, se realizan algunas consideraciones finales y se muestran los desafíos encontrados para las redes estudiadas y lo que enseñan sobre el tema.

\section{Redes y redes económicas solidarias}

Para hablar de redes, es necesario presentarlas en sus diferentes alternativas de abordaje. Las redes para Granovetter (1990) están compuestas por un conjunto de relaciones o lazos entre los actores. Más específicamente, este autor define redes sociales como un conjunto de núcleos o de actores ligados por relaciones sociales o lazos de tipos específicos, de modo que un lazo o relación entre dos actores tienen fuerza y contenido. El contenido incluye información, intereses compartidos $y$, por lo menos, algún nivel de confianza.

Las redes son concebidas, también, como formas de gobernabilidad. Según Piore y Sabel (1984), a partir de la década de 1970, con la saturación del mercado de productos en masa, las empresas comienzan a flexibilizar la producción y a trabajar en redes. Esa producción desarrolla capacidades tales como conseguir integrar más proveedores y distribuidores por medio de procesos de externalización y alterar sus productos en función de las nuevas tecnologías, entre otras. Las nuevas lógicas de producción, en un ambiente competitivo, dependen del trabajo en red entre las firmas que componen la cadena de producción y distribución.

Se busca diferenciar dos concepciones de red: una, cuyas motivaciones se concentran en la garantía de más

\footnotetext{
1 Este artículo hace parte de la disertación de maestría, defendida en noviembre del 2011 en el Departamento de Sociología de la Universidad de Brasilia. Para ver la disertación completa en portugues, ingrese a: http://repositorio.unb.br/bitstream/10482/11556/1/2011_IvetteTatianaCastillaCarrascal.pdf
}

ganancias privadas (de individuos o empresas capitalistas), envolviendo explotación de recursos y fuerza de trabajo conforme una lógica o estructura jerárquica. Y otra, cuyas motivaciones se centran en la solidaridad entre individuos organizados en grupos descentralizados, los cuales buscan asegurar ganancias colectivas amparados en estrategias de producción y distribución horizontales.

En relación con esta última, Mance (2008) afirma que una red es una articulación entre diversas unidades que, a través de conexiones, intercambian elementos entre sí, fortaleciéndose recíprocamente y tendiendo a multiplicarse en nuevas unidades. A su vez, estas unidades refuerzan todo el conjunto $\mathrm{y}$, al mismo tiempo, este nuevamente las fortalece, permitiéndoles expandirse en nuevas unidades o mantenerse en equilibrio sustentable.

Según França y Cunha (2009), las redes tienen dos objetivos principales: permitir la sostenibilidad de los emprendimientos y fortalecer el potencial endógeno de un territorio sobre su capacidad de promover su propio proceso de desarrollo. Además de eso, las redes inducen la constitución de circuitos propios de comercialización y producción, y crean esa nueva modalidad de regulación económica, lo que supone otro modo de funcionamiento de la economía real. Los contratos y acuerdos son establecidos con base en principios, valores y reglas que van más allá de los imperativos de rentabilidad económica de la actividad.

Las redes tienen algunos principios que las guían. Estos principios propios de las redes solidarias son enumerados por Mance (2008), tal como se muestra en la tabla 1, donde están sintetizados.

Las redes, según Mance (2008), están organizadas en núcleos, de manera que cada núcleo representa una unidad y cada hilo, un canal por el cual esas unidades se articulan a través de diversos flujos. Los tipos de flujos son de materiales (compras y ventas de insumos o productos que circulan por la red), de información (divulgación de productos y servicios, transferencias de tecnología), y de valores (son los recursos que circulan por la red; flujos económicos que hacen viables los procesos formativos, informativos, organizativos y políticos).

Tygel (2010) describe los flujos que cada red tiene, en una perspectiva que se aproxima más a los emprendimientos económicos solidarios (EES), como se muestra en la tabla 2. 
Tabla 1

Principios de las redes

\begin{tabular}{ll}
\hline \multicolumn{1}{c}{ Principios } & \multicolumn{1}{c}{ Descripción } \\
\hline Sistema abierto & $\begin{array}{l}\text { Se autocrea, conecta grupos y realiza la } \\
\text { articulación entre ellos y fortalece cada } \\
\text { grupo en particular por los intercambios } \\
\text { que ocurren. }\end{array}$ \\
\hline Cada unidad de la red es intensiva con el \\
fin de alcanzar e incluir un mayor número \\
de personas en el lugar donde actúa. Esto \\
puede significar el surgimiento de otros \\
pequeños nódulos en una región específ- \\
ca, articulados entre sí y conectado a la red \\
en general.
\end{tabular}

Nota. Adaptado de La revolución de las redes, por E. Mance, 2008.

Los objetivos de los estudios sobre redes son amplios. Hay estudios que apuntan a explicar la proliferación de industrias y multinacionales organizadas en red, sus impactos y formas de organización y gestión. Hay otros cuyo foco es el fortalecimiento de redes y espacios de comercialización alternativos, en los cuales pequeños productores consiguen participar de intercambios comerciales e integrarse con otros actores. Para ejemplificar el primer caso, Powell (1990) afirma que existen en el sistema capitalista muchas empresas en forma de red, las cuales se constituyen como industrias altamente competitivas. Esas proliferan en red porque existen condiciones favorables tales como la demanda por velocidad, el conocimiento y la confianza;
Tabla 2

Flujos de las redes

\begin{tabular}{cl}
\hline \multicolumn{1}{c}{ Flujos } & \multicolumn{1}{c}{ Descripción } \\
\hline \multirow{5}{*}{ Saberes } & $\begin{array}{l}\text { Representa aquellos conocimientos que existen } \\
\text { al interior de las redes, los cuales contemplan las } \\
\text { diferentes historias de vida, formas tradicionales de } \\
\text { producción y de comercialización. }\end{array}$ \\
\hline \multirow{5}{*}{ Pste flujo se relaciona con un cierto ejercicio demo- } \\
crático dentro de las redes, esto es, con la participa- \\
ción de los integrantes del grupo en las decisiones \\
internas. Deben ser observados los puntos en los \\
que está concentrado el poder para las tomadas de \\
decisión. \\
Posibilidad de participación de todos los integrantes \\
del grupo en los procesos de toma de decisiones de \\
los EEs o de la red.
\end{tabular}

Nota. Adaptación de "Fluxos e informações na economía solidária: um novo olhar sobre as práticas", por D. Tygel, 2010, en Cartilha Economía Solidária, 1, IBASE.

son más adaptables a las mudanzas ya que tienen más habilidad para interpretar nuevas informaciones y traducirlas en nuevos productos.

A fin de ilustrar el segundo caso - en el cual se ve el mercado en red desde las organizaciones de pequeños productores-, Azevedo (2010) afirma que los mercados solidarios se configuran en redes locales que articulan diversos instrumentos económicos, sociales y educativos. Estos instrumentos serían tan diversos como: cooperativas locales, sistemas de educación no formal, formación de personas adultas y pequeñas tiendas, entre otros. Conceptualmente, la discusión sobre el mercado solidario tiene su origen en las redes colaborativas y solidarias. Entre sus aspectos positivos, está 
su contribución al desarrollo sostenible, a la generación de ingresos y la reducción de la jornada laboral, el aumento del ahorro interno, la distribución de la riqueza y la asociación libre de empresas (Mance, 1999).

Según Melo-Lisboa (2004), no es posible entender la persistencia de las relaciones económicas del mercado como una simple victoria del laissez-faire, a pesar de la gran transformación que llevó a los mercados a continuar siendo un espacio social, esto es, la forma de socialización en la cual se producen encuentros e intercambio de informaciones, y no solo transacciones con una orientación utilitaria.

En la reflexión de Melo-Lisboa, el mercado podría ser el locus de la estructuración de la sociedad moderna, el espacio de bienes, el lugar de la distribución y el consumo. La superación de la sociedad de mercado no quiere decir la ausencia de mercados. Basado en Polanyi (2000), el autor plantea la cuestión de pensar la diferencia entre la sociedad de mercado, por un lado, y la sociedad con mercado, por otro.

El primer análisis de un mercado solidario apunta al campo empírico de las redes, en el cual las observaciones llevan a creer que las redes de economía solidaria fortalecen su identidad a través de varias prácticas, entre las cuales están las relacionadas con los principios del comercio justo y solidario. Fortalecen los vínculos sociales entre los grupos y se establecen los acuerdos comerciales sobre la base de principios, lo cual promovería la coordinación entre los actores. También, con el fin de llegar a la sostenibilidad económica de las redes, sería necesario desarrollar algunas prácticas de planeación, implementación y control del flujo de las redes, y, por lo tanto, extender las posibilidades de aumentar sus ingresos y mejorar la comercialización, así como crear $\mathrm{y}$ fortalecer espacios alternativos.

\section{Organización en red}

La globalización ha sido caracterizada por una progresiva organización de cadenas productivas a nivel internacional. Según Parreiras (2007), para que las poblaciones pobres tengan una inserción económica sostenible y un acceso a mayores ganancias, sería necesario una acción económica amparada en una participación organizada en cadenas productivas. Caso contrario, los productores están condenados a la marginación o a una vida de subsistencia, perpetuando la pobreza.

De igual forma, según Parreiras (2007), son necesarias estrategias de participación en la organización de redes y cadenas, así como el desarrollo de productos de calidad con el fin de garantizar que valores sean agregados con la industrialización y comercialización. Este sería un camino para el enfrentamiento de la pobreza, que debe estar combinado, por fin, con acciones políticas nacionales e internacionales en el ámbito de las instituciones multilaterales y en el fortalecimiento de iniciativas como el comercio justo.

Por otro lado, según Azevedo (2010), las redes locales de economía solidaria permiten el desarrollo de una relación de distribución y proximidad entre productores(as), prestadores(as) de servicios y consumidores(as) locales, de manera que son fundamentales para la sostenibilidad de las comunidades y de los recursos endógenos disponibles. Los productos y saberes locales aumentan su valor social al ser aprovechados y distribuidos dentro de la propia comunidad de donde provienen, pues son los productos locales los que mantienen el mundo rural vivo.

Según Mance (2008), las redes locales, teniendo como base principios organizativos, actúan en el sentido de responder necesidades inmediatas de la población por trabajo, mejoría del consumo, educación, reafirmación de la dignidad humana y de su derecho al buen-vivir; simultáneamente combaten las estructuras de explotación y dominación responsables por la pobreza y exclusión.

Sonnino y Marsden (2005) afirman que la participación en las redes alternativas corresponde a la conexión de productores, consumidores y demás actores de las redes a las posibilidades de diversificación productiva, determinada antes por los tiempos relativos a la estacionalidad de las culturas (producciones) y al manejo por la demanda pautada en el mercado, de escalas mayores y tiempos reducidos.

Las redes alternativas de producción y consumo de alimentos, a partir del momento que incorporan en sí mercados alternativos, tales como ferias y cooperativas de consumidores - mercados que permiten la diversidad y la escala características de las pequeñas producciones-, así estén asociadas, presentan autonomía en relación con las cadenas convencionales, lo cual confiere este atributo a los procesos de producción, libre asociación, comercialización y consumo de productos agroalimentarios.

Las anteriores son algunas afirmaciones del por qué la conformación de redes puede mejorar la calidad de vida de comunidades y grupos. Las redes de economía solidaria son muchas veces consideradas con un carácter duplo, algunas predominantemente políticas 
y otras predominantemente productivas o comerciales. Las "redes políticas" dan mayor énfasis a la articulación de sus actores, a fin de hacer incidencia en las políticas públicas y así representar los intereses de los emprendimientos. Es también objetivo de muchas de esas redes construir una identidad en la defensa del proyecto político del movimiento, lo cual lleva a la construcción de representaciones con el fin de reforzarla. Las redes políticas favorecen la cooperación y la intercooperación entre movimientos sociales y organizaciones de diversa naturaleza, así como promueven la organización del movimiento para incidir en procesos públicos y hacer visible la economía solidaria.

Las redes que enfatizan la actividad productiva buscan la construcción de cadenas, en las cuales se realizan funciones de producción, comercialización y consumo de forma integrada o complementaria. La organización en redes puede llegar a facilitar la comercialización de los productos de pequeños productores en diversos espacios alternativos. Estos pueden ser desde ferias hasta grupos de consumidores organizados, con fines de todo tipo: desde comprar directamente sus materias primas para la elaboración de los productos hasta comercializar en grupo.

La autogestión en red permite así repensar el proceso productivo y construir cadenas de producción e intercambio de informaciones y conocimientos, lo cual potencializa otra forma de organización social y económica. En la economía solidaria se valoriza la comunicación en redes horizontales y la construcción de cadenas productivas.

\section{Las redes económicas solidarias en Brasil}

En Brasil se han organizado emprendimientos en cadenas productivas y redes solidarias, con el fin de superar algunos obstáculos encontrados en la comercialización y producción. Algunas redes son incentivadas por el gobierno y entidades de apoyo, otras, por la iniciativa de los propios trabajadores. La producción en colectivos, o la compra de materias primas o insumos, así como la creación de espacios de comercialización, son algunos de los motivos por los que las redes se unen.

Todas las redes de economía solidaria son en principio redes sociales, ya que envuelven un gran número de actores cuyos vínculos exigen un compromiso colectivo más amplio. Generalmente, las redes son formadas por una gran diversidad de actores. En Brasil, se encuentran organizaciones no gubernamentales, organizaciones de pequeños productores, cooperativas y asociaciones (rurales y urbanas), tiendas de comercio justo, grupos de agricultores orgánicos y agroecológicos, sectores académicos, sectores de la Iglesia, sindicatos o centrales sindicales, entre otros.

Ha sido una afirmación del movimiento de economía solidaria brasilero (según el Informe de la IV Plenaria) que los emprendimientos solidarios sean motores de desarrollo local, solidario y sustentable. Esto indica la responsabilidad e importancia de los ejes de producción, comercialización y consumo solidarios, así como de la estrategia de organización y articulación en redes y cadenas solidarias.

Esa deliberación es una de las más importantes del movimiento, habiendo sido reforzada en el informe final de la II Conferencia Nacional de Economía Solidaria:

La estrategia nacional de comercialización solidaria busca el incentivo a la Comercialización Solidaria, al Comercio justo y solidario y al Consumo responsable. Para eso, necesariamente, deberá: incentivar y financiar la creación de espacios de comercialización solidarios permanentes y centros públicos de economía solidaria territoriales; apoyar la constitución y construcción de redes y cadenas solidarias de producción y de agro industrialización, de comercialización de logística y de consumo solidario; identificar cadenas productivas étnicas; implantar procesos de certificación participativa y el sello de la economía solidaria; promover la identidad visual y territorial de los productos y servicios; promover la formación/asesoramiento técnico continuo y sistemático a la comercialización; promover el consumo responsable; y fomentar la priorización de productos y servicios de la Economía Solidaria en las compras institucionales en todas las esferas, modificando la ley $8.666 / 93$ y expandiendo las adquisiciones para cualquier producto o servicio de la economía solidaria. Tales acciones deben estar articuladas y en consonancia con los principios, regulaciones y criterios establecidos en el Sistema Nacional de Comercio Justo y Solidario (Secretaria Nacional de Economia Solidária, 2010, artículo 98).

En América Latina, el principal eje que las une es el comercio justo. En los últimos años, numerosas organizaciones en el continente se dedican al desarrollo de mercados locales y regionales. El lugar del comercio justo como elemento activo de la economía solidaria está fuertemente reafirmado por sus socios suramericanos que ya trabajan en redes de manera amplia con 
sus homólogos, asociaciones de consumidores, sindicatos, organizaciones de defensa de los derechos humanos y del medio ambiente, etc.

De hecho, las acciones de incidencia política emprendidas en el marco de estas otras redes más amplias tienen más impacto, tanto a nivel político como a nivel público, y este es uno de los objetivos fundamentales del comercio justo: la implementación de campañas de sensibilización en escalas nacional e internacional.

\section{Las redes objeto de análisis}

Las redes objeto de análisis son cinco: Central do Cerrado, Rede Bodega, Acs Amazonia, Rede Bodega y Rede Ecovida, cada una localizada geográficamente en regiones diferentes. Estas redes son de diferente naturaleza, integradas por diferentes actores y conexiones, pero hacen parte de un mercado solidario o de un mercado en red. Fueron escogidas con base en la evidencia según la cual representan diferentes realidades de todo el territorio nacional. Estas redes han sido referencia para otras por su forma de organización y articulación en espacios nacionales, regionales, y por ser reconocidas por otras redes y por los propios actores de la economía solidaria.

Lo que se presenta a continuación son las descripciones de las redes realizadas con base en la metodología del Actor-Red, propuesta por Bruno Latour (2008). Esta metodología propone describir los flujos, las conexiones, los actores y los efectos que esos flujos tienen al interior de las redes y fuera de ellas de manera detallada. Por otro lado, antes de llegar a estas descripciones, se recolectaron los datos e informaciones mediante entrevistas semiestructuradas aplicadas en el trabajo de campo.

Con base en la metodología mencionada, se identifican los diferentes tipos de flujos que traspasan las redes: sean estos de productos, de saberes, de valores, económicos, entre otros. Se observan los principales desafíos que se presentan para estos grupos, así como algunos de los impactos que los emprendimientos económicos solidarios experimentan al hacer parte de redes alternativas de producción y comercialización.

\section{Central do Cerrado}

La Central do Cerrado, localizada en la región centrooeste, reúne emprendimientos de estados de Maranhão, Pará, Tocantins, Piauí, Goiás, Mato Groso, Minas Gerais y Mato Grosso do Sul, y su sede se encuentra en
Brasilia D. F. La Central se define como una iniciativa que desarrolla actividades de apoyo a la comercialización de 35 organizaciones comunitarias, las cuales producen a partir del uso sostenible de la biodiversidad del Cerrado. Es una central de cooperativas y funciona como un puente entre productores comunitarios y consumidores, ofreciendo productos procesados por agricultores familiares y comunidades tradicionales en el bioma Cerrado.

Desde el 2002, el Instituto Sociedad Población y Naturaleza (ISPN) comienza con la discusión de una comercialización de los productos del bioma Cerrado. Mediante el proyecto de PPECOS ${ }^{2}$, se elabora un catálogo que busca facilitar el acceso a los productos de los grupos. Los productores comenzaron a percibir que podían juntarse para las ferias y participar así de más espacios de comercialización. Fue en ese momento que se montó una oficina para tal fin. Los emprendimientos ya tenían su mercado local construido, pero cuando se agotaban las posibilidades con los conocidos comenzaban a tener problemas.

En aquel tiempo, 19 organizaciones constituían la central de manera informal. El pPECos ayuda a estructurar la central de comercialización, al realizar reuniones cuyo fin era trazar las estrategias de funcionamiento, los compromisos de cada uno y, así, un reglamento interno. En el 2010, se formalizan y en febrero se realiza la asamblea de fundación.

Actualmente, además de promover la divulgación e inserción de los productos comunitarios de uso sustentable del Cerrado en los mercados locales, regionales e internacionales, la Central sirve también como centro de propagación de informaciones, intercambio y apoyo técnico para las comunidades en la mejoría de sus procesos productivos, organizacionales y de gestión.

La mayoría de materias primas e insumos usados para realizar los productos vienen del Bioma Cerrado. Tienen un estimado de las capacidades de producción de cada grupo, actualizándolas siempre dependiendo

\footnotetext{
2 El Programa de Pequeños Proyectos Ecosociales (PPP-ECos) fue creado para apoyar proyectos de organizaciones no-gubernamentales y de base comunitaria que desarrollen acciones, las cuales generan impactos ambientales globales positivos, combinados con el uso sostenible de la biodiversidad. Es coordinado técnico-administrativamente por el Instituto Sociedad, Población y Naturaleza (ISPN). El PPP-ECos es uno de los pocos programas en Brasil que direcionan su apoyo exclusivamente para el bioma Cerrado y sus áreas de transición con la Amazonía, el Pantanal, la Caatinga y la Mata Atlantica. Ver http://www.ispn.org.br/projetos/ ppp-ecos-programa-pequenos-projetos-ecossociais/
} 
de las cosechas. Los espacios de comercialización donde se realizan las ventas son ferias locales, nacionales, restaurantes y tiendas de productos naturales. Algunos grupos, como la Cooperativa Grande Sertão, venden al Programa de Adquisición de Alimentos (PAA); otros realizan ventas directas a las alcaldías municipales. La central participa de muchas ferias convocadas por entidades del gobierno, diversas ONG, y ferias organizadas por los propios emprendimientos de alguna ciudad, entre otros espacios. Además de las ferias y eventos, venden de forma directa en su espacio físico, siendo este un lugar de representación y distribución. La comercialización al por mayor se ha realizado para algunos restaurantes de Río de Janeiro y São Paulo; también han sido realizadas alianzas con el movimiento de Slow Food para la diversificación de sus productos.

En relación con el flujo de saberes, lo más importante es el intercambio entre productores. La central ha organizado algunos intercambios a fin de tratar temas específicos y para fortalecer algunos grupos que necesitan de apoyo; los productores intercambian saberes y conocimientos sobre el Cerrado y sobre diversas formas de solucionar problemas, sea en la producción, en la comercialización o en el consumo.

\section{Rede de agroecología Ecovida}

Se define como una red de agricultores familiares, técnicos y consumidores reunidos en asociaciones, cooperativas y grupos informales que, con pequeñas agroindustrias, comerciantes ecológicos y personas comprometidas con el desarrollo de la agroecología, apuntan para algunos objetivos. Estos objetivos son: desarrollar y multiplicar iniciativas de agroecología; estimular el trabajo asociativo en la producción y en el consumo de productos ecológicos; aproximar de forma solidaria agricultores y consumidores; estimular el intercambio, el rescate y la valorización del saber popular; y tener una marca y un sello que expresen el proceso, el compromiso y la calidad. El número de familias aproximadas que hacen parte de la Red Ecovida es de 2100. Cuenta con 21 núcleos regionales, más de 220 organizaciones de agricultores, 25 oNG, 10 cooperativas de consumidores y decenas de pequeñas agroindustrias e iniciativas de comercialización. Comprende más de 170 municipios y estimula la comercialización directa a través de aproximadamente 120 ferias ecológicas en toda la región sur.

La red se encuentra en la región sur de Brasil, en los estados de Santa Catarina, Paraná y Rio Grande do Sul. La Red Ecovida es reconocida por ser una Red de
Certificación Socioparticipativa. Sin embargo, antes de la certificación los productores del sur del país ya comercializaban en ferias y pequeñas tiendas su producción ecológica, desde mucho antes de formarse la red Ecovida. Si bien esta nace en 1998, su historia se remonta al inicio del movimiento agroecológico en Brasil. En los años ochenta, la organización del movimiento agroecológico hizo un rescate y difusión de tecnologías alternativas y comenzaron con ferias en los mercados locales, grupos y cooperativas de consumo y puntos de comercialización. En esta década se tuvo una propuesta más política, desde un enfoque más técnico a cuestiones sociales de la producción.

A principios de la década de 1990, se experimenta el surgimiento de múltiples iniciativas en el campo de la producción agroecológica, con el fin de minimizar o frenar las consecuencias negativas de la "modernización conservadora”. En esta década, surge el mercado orgánico a nivel internacional y las primeras reglamentación orgánicas nacionales.

Cuando la Red Ecovida comienza formalmente en 1998, ya los grupos producían vegetales, cereales, frutas, huevos, jugos, mermeladas, frijol, hortalizas, maní, maíz (diversas variedades), especies, miel, leche y procesados de carnes y embutidos, entre otros productos.

En 1994, sale la primera legislación europea sobre productos orgánicos, y tienen inicio en Brasil los primeros debates para construir un marco legal. En aquel momento, el Ministerio de Agricultura, Pecuaria y Abastecimiento (MAPA) llama a un grupo de personas para debatir el tema, y solo en 1999 fue lanzada una instrucción normativa (IN 07/99) que definía el sistema orgánico de producción, la cual postulaba la exigencia de reconocimiento y comercialización de productos orgánicos.

En el 2001, sale la norma IN16, en la cual se acreditan las certificadoras, y en el 2002, se realiza el Encuentro Nacional de Ecologista (ENA), así como es creado el Grupo de Agricultura Orgánica (GAO), que participa en los debates para la propuesta de Ley 10.831 , hecha en el 2003.

Los Sistemas Participativos de Garantía (SPG) surgen como una resistencia frente al mercado de certificación orgánica que se institucionalizó con la Ley 10.831 . El movimiento agroecológico llegó a un consenso según el cual la certificación sería prevista, pero se adecuaría metodológicamente a la realidad donde se desarrollaría y no sería exigido el sello en la venta directa. El consenso permitió que se desarrollaran los criterios relativos a la agricultura orgánica, conceptos, 
características, y los mecanismos de los sistemas de garantía: la certificación ${ }^{3}$ "por auditoría" y de generación de credibilidad ${ }^{4}$ o certificación participativa (Sistema Participativo de Garantía, SPG).

La mayoría de los grupos que comercializa productos in natura en ferias agroecológicas no utiliza el sello, pues los productos no son embalados y la forma de comercialización no exige certificación, a excepción de los grupos que comercializan en ferias donde los demás productos son convencionales y precisan de una forma de diferenciación. La certificación es exigida por supermercados o mercados institucionales. Uno de esos mercados institucionales es el programa del gobierno federal llamado Programa de Adquisición de Alimentos (PAA), en el cual algunos grupos de la red proveen alimentos.

\section{Justa Trama}

Justa Trama es una cadena productiva que tiene entre sus actividades económicas la plantación de algodón orgánico, el beneficio, la confección y la comercialización de piezas de tejidos. Es una central de cooperativas que cuenta con una asociación y cuatro cooperativas. Aproximadamente son 700 asociados, organizados en cooperativas y asociaciones productivas. La cadena comprende seis estados: Ceará, Rondônia, São Paulo, Minas Gerais, Santa Catarina y Rio Grande do Sul.

La historia de Justa Trama comienza en el 2004 cuando para el Foro Social Mundial del 2005 se hicieron unos pedidos de bolsos. Unas 70000 unidades fueron divididas entre la Cooperativa Univens, la Cooperativa Fio Nobre y otras cooperativas de la región para cumplir con el desafío. Después de esto, entre las cooperativas que participaron del pedido, se plantearon algunas inquietudes de continuar trabajando como lo habían hecho. Así comenzaron las discusiones para formar una cadena que integrara la producción desde la plantación del algodón orgánico, hasta la elaboración de las piezas.

\footnotetext{
3 La certificación es un procedimiento de evaluación de la conformidad, en el cual un organismo de tercera parte (independiente de producción y del consumo), certifica por escrito que determinado producto, proceso o servicio está de acuerdo con las normas o reglamentos preestablecidos.

4 La generación de credibilidad es un proceso de garantía que se da a través de las relaciones entre productores y consumidores, o a través de la organización de base y de las relaciones en red, siendo una evaluación de la conformidad sobre control social o certificación participativa.
}

Las personas que hacen parte de Justa Trama ya pertenecían a cooperativas hace algún tiempo y, desde entonces, realizaban conferencias en escuelas y espacios de formación con el fin de enseñar lo que era autogestión, y hacían también parte de las discusiones del movimiento de la economía solidaria.

En la cadena, todos los años, en el periodo de septiembre a diciembre, se realizaba una sensibilización a los agricultores para la plantación agroecológica, realizada por la Asociación de los Agricultores de Algodón (ADEC), en Ceará, junto con Esplar (entidad de asesoría). Ellos hacen una planificación de la plantación, y determinan la cantidad prevista, lo que plantarán, quién plantará y para quién van a vender.

Una vez hecha esa planificación, los agricultores se inscriben, y ADEC y Esplar realizan un trabajo de acompañamiento con sus técnicos. El acompañamiento es realizado a partir de abril que comienza la plantación. De junio a septiembre es el período de recolección y los agricultores de los municipios recogen, almacenan, y envían para ADEC. ADEC recibe y paga el algodón en rama, lo almacena y comienza el procesamiento, que es limpiar el algodón y retirar la pluma.

Al final, generalmente en agosto o septiembre, se envía para la cooperativa textil. Entra a la línea de producción, salen hilos y luego a las máquinas para producción de las telas. Después va para beneficio y sigue para las cooperativas Univens y Fio Nobre.

El almacenamiento es en rollos con cuidados necesarios o en estantes para ventas en las tiendas o espacios de emprendimientos asociados. La sede "oficial" queda dentro de la Cooperativa Univens, en Porto Alegre. Además, Justa Trama tiene cinco puntos de comercialización. Uno de estos se encuentra en el Mercado Municipal de Porto Alegre, otros son tiendas de los asociados, centros públicos y tiendas de economía solidaria. Tienen alianzas internacionales, especialmente con España e Italia, países que consumen, divulgan y venden piezas de Justa Trama.

\section{Rede Bodega}

En la Región Nordeste, la Rede Bodega está compuesta por diferentes tiendas situadas en las ciudades de Fortaleza, Tianguá, Sobral, Aracati y la Prainha do Canto Verde, todas en el Estado de Ceará. Las bodegas son cooperativas de producción y comercialización y trabajan con grupos de agricultores familiares y artesanos. Realizan intercambios de productos, participan de 
actividades de formación, de intercambio de saberes e informaciones entre las comunidades, y en espacios de planificación de las actividades.

Las bodegas son cooperativas de producción y comercialización, las cuales trabajan con grupos de agricultores familiares y artesanos. La Rede Bodega inició en el 2004 con el proyecto "Bodegas: espacios agroecológicos y solidarios”. El proyecto contempló la creación de dos bodegas, acompañadas por la Pastoral Social de la Diócesis de Tianguá y Cáritas Diocesana de Limoeiro do Norte, y fue apoyado por Cáritas Regional y Cáritas Diocesana de Limoeiro, y la Catholique Relief Service (CRS), la cual financió la estructuración de los dos espacios y la contratación de agentes de ventas. Así comenzaron las Bodegas Nordeste Vivo y Solidario y Bodega do Povo.

En el 2007, nace Budegama, con un proyecto en alianza con el Banco do Nordeste BNB. Esta experiencia estaba constituida por diez mujeres artesanas que ya se encontraban organizadas en la Asociación de Mujeres en Acción (AMA). En el 2008, nace la cuarta Bodega, Budega Arcos, la cual inició con el apoyo de la diócesis de Sobral y otras entidades.

Cada una de las bodegas tiene su espacio de comercialización. La Bodega Nordeste Vivo y Solidario tiene dos espacios físicos para la comercialización de productos artesanales y agroecológicos, uno en Aracati y otro en la Prainha do Canto Verde, en Beberibe. $\mathrm{Bu}-$ degama tiene un espacio de comercialización en la ciudad de Fortaleza, la Bodega Arcos, la cual se encuentra en Sobral y tiene su espacio de comercialización en alianza con la terminal del municipio; la Bodega do Povo tiene un espacio en el municipio de Tianguá.

Las bodegas realizan intercambios de productos, participan de actividades de formación, de intercambio de saberes e informaciones entre las comunidades, así como espacios de planificación de sus actividades. Todas tienen fondos rotativos de apoyo a la producción y de apoyo a la comercialización. Los de apoyo a la producción están encargados de potenciar las actividades productivas de los grupos mediante recursos financieros. Estos fondos pueden ser fijos (no devolutivos) y rotativos. Los fijos tienen una destinación para compras de equipamientos, máquinas e infraestructura para los grupos. Los rotativos son para compra de insumos y son todos devolutivos.

Por otro lado, los fondos rotativos de apoyo a la comercialización están encargados de potenciar la comercialización de los grupos mediante recursos financieros que también pueden ser para fondos fijos o rotativos. Los fijos (no retornan) son para compras de equipamientos e infraestructura para las bodegas. Los fondos rotativos sirven para dar un adelanto del 50\% del valor de los productos disponibles en las bodegas que van a ser comercializados. Los recursos que retornan vuelven a los fondos rotativos, de manera que permitan el acceso de esos recursos a otros grupos.

\section{Asociación de certificación socioparticipativa de la Amazonia}

La Rede ACs Amazonia está en la región norte del país, en el Estado de Acre, municipio de Rio Branco. La red realiza visitas a las comunidades de agricultores con técnicos agrícolas para el monitoreo de las áreas. La ACS, desde diciembre del 2002, se encarga de revitalizar la Feria de Productos Orgánicos, pero es mucho más una entidad de asesoría que una entidad de apoyo a la comercialización.

Comienza desde el 2001, cuando sale una propuesta de gobierno para fomentar una cadena productiva agroforestal. Es en el 2003, en la realización del II Workshop de Certificación participativa en Red (Rio Branco-Acre), que comienzan formalmente. Acs hace parte de Faces do Brasil, del Foro Brasilero de SPG y de la Cámara Técnica de agricultura orgánica, entre otros.

La Feria de Orgánicos está localizada frente al mercado de alimentos del municipio. El punto de ventas es estratégico, pues muchas personas comienzan a conocer los productos orgánicos cuando van pasando para la otra feria. La feria existe desde 1998, pero había decaído, y desde el 2002 es que ACs se encargó de revitalizarla. Actualmente comercializan cuatro grupos: Moreno Maia, Wilson Pinheiro, Bem Fica y Humaitá.

Cada grupo tiene su reglamento interno para producción agroecológica, el cual fue desarrollado con ayuda de los técnicos de la ACs. Incorpora algunos otros factores de organización interna.

\section{Consideraciones finales}

En la investigación que resultó de la Maestría en Sociología, se experimentó de manera próxima la realidad de los emprendimientos que hacen parte de redes alternativas de producción y comercialización. El incentivo a la conformación y fortalecimiento de redes ha sido prioridad para el movimiento de economía solidaria en Brasil, así como para las políticas relacionadas con el tema. Esto ha sido reforzado tanto en la 
v Plenaria de Economía Solidaria ${ }^{5}$, como en la II Conferencia Nacional de Economía Solidaria ${ }^{6}$.

La economía solidaria y el comercio justo han sido alternativas para miles de pequeños productores excluidos del mercado formal, altamente regulado. La diversidad de actores que participan de este movimiento es amplia, y cada vez más se unen en diversos tipos de redes con el fin de participar de espacios de comercialización y producción, o espacios de incidencia política.

A pesar de las diferencias entre las redes investigadas, comenzando por el contexto geográfico donde se encuentran, se observan semejanzas en los impactos que los emprendimientos experimentaron. Entre estos se encuentran: la creación de lazos y relaciones de confianza entre los miembros de la red; fortalecimiento de una identidad; valorización de los jóvenes por el trabajo en el campo; intercambio de experiencias que permitió a los grupos acceder a más conocimientos sobre relación con los consumidores, espacios directos de venta, organización de los grupos, alianzas, autosostenibilidad, finanzas solidarias, conversión agroecológica, integración entre productores y consumidores, relaciones más horizontales de trabajo y fortalecimiento de la participación en la toma de decisiones en los grupos, entre otras.

Estas cinco redes tienen el desafío de la sostenibilidad económica, y la no dependencia de alianzas o recursos de proyectos de cooperación. De igual forma, el desafío de la autogestión y mejor organización al interior de los grupos y a la hora de comercializar; pensar en nuevas alternativas y estrategias comerciales, las cuales fortalezcan la identidad de los productos y de los grupos; sostenibilidad en la producción, criterios de respeto a la naturaleza y solidaridad entre los miembros de un mismo grupo y entre grupos diferentes.

Con respecto a la certificación de productos, queda el desafío de valorar los procesos mucho más que los

\footnotetext{
5 En la Carta Política Final de la v Plenaria, se colocó como pauta para el Estado Brasilero: implantación de políticas públicas, organizadas a partir del territorio, que fortalezcan los emprendimientos económicos solidarios, priorizando el apoyo a su financiamiento, la comercialización solidaria y el estímulo a su organización en redes y cadenas económico-solidarias.

6 Artículo 98. Relato II CONAEs: "La estrategia nacional de comercialización solidaria busca incentivar la Comercialización Solidaria, el Comercio justo y solidario y el Consumo responsable. Para esto, necesariamente, deberá: promocionar y financiar la creación de espacios de comercialización solidarios permanentes y centros públicos de economía solidaria territoriales; apoyar la constitución y construcción de redes y cadenas solidarias de producción y de agro industrialización, de comercialización, de logística y de consumo solidario (...)”.
}

productos, para no caer en una "cultura del sello". En algunas ocasiones, la certificación deja de ser un eslabón de relación entre producción y consumo y se convierte en una mercancía que busca ampliar el nicho de mercado a fin de ampliar sus beneficios. El problema de ese enfoque es que amenaza la existencia de redes sociales tan necesarias.

Otro desafío importante es el reconocimiento de los sistemas participativos de garantía a nivel internacional, y la posibilidad de que los productos garantizados por los SPG puedan transitar entre los diferentes países. Las certificadoras convencionales reconocen la calidad de la certificación participativa, y se tiene un entendimiento de que el proceso de certificación participativa es válido solo para el mercado local, de modo que no puede ser utilizado donde existe un distanciamiento entre el productor y el consumidor.

El fortalecimiento de espacios de participación colectiva y la toma de decisión es un desafío común de todas las redes investigadas. La superación de este desafío permitiría fortalecer vínculos y realizar una planificación de la red, en la cual se integren las necesidades de todos sus actores. Es importante también el monitoreo de las acciones establecidas como prioridades para el fortalecimiento de las redes.

La promoción de intercambios de experiencias entre los diferentes grupos es un desafío. A pesar de que todas las redes ya realizan esos intercambios, se identifica que estos potencian el acceso al conocimiento e información sobre cómo lidiar con los problemas comunes.

Como resultado de la investigación, además de las especificidades de las redes, se evidencia que esas y otras redes, junto con cientos de emprendimientos en Brasil, comercializan en lo que puede ser denominado mercado solidario, los espacios creados específicamente para la comercialización de productos de la economía solidaria y del comercio justo y solidario. Las estrategias de comercialización más usadas son la participación en ferias permanentes, tiendas, entrega directa al consumidor y otro tipo de ferias específicas.

La mayoría de los emprendimientos que hacen parte de las redes buscan actuar, bien sea en una o varias de las anteriores opciones, bien sea en mercados que valorizan el carácter cultural de los productos y en los cuales los consumidores prefieren los productos por su origen, asociado al proceso de producción, por ejemplo, al carácter agroecológico. 


\section{Referencias}

Azevedo, R. (2010). O papel das redes locais nas economias solidárias - O caso dos mercados solidários da Granja do Ulmeiro. Universidade de Coimbra.

França Filho, G. C., Cunha, E. V. (2009). Incubação de redes de economia solidária. En A. D. Cattani, J. Laville e P. Hespanhao (Eds.), Dicionário Internacional da Outra economia. Coimbra: Altamira.

Granovetter, M. (1990). The Old and the New Economic Sociology. En R. Friedland y A. F. Robertson, Beyond the Market Place (pp. 81-112). Aldine de Gruyer.

Latour, B. (2008). Reensamblar lo social: una introducción a la teoría del actor-red. Buenos Aires: Ediciones Manantial.

Mance, E. (1999). A Revolução das Redes: a colaboração solidária como uma alternativa pós capitalista à globalização atual. Petrópolis: Vozes.

Mance, E. (2008). La revolución de las redes: la colaboración solidaria como una alternativa post-capitalista a la globalización actual. México: Editorial Ítaca.

Melo-Lisboa, A. (2004). Mercado Solidário. En A. D. Cattani (Ed.), La Otra Economía (pp. 293-305). Buenos Aires: ungs-Fund. Osde-Altamira.
Parreiras, L. E. (2007). Negócios Solidários em Cadeias Produtivas. Protagonismo coletivo e desenvolvimento sustentável. Rio de Janeiro: IPEA/ANPEC/FBB.

Piore, M. y Sabel, C. F. (1984). The Second Industrial Divide: Possibilities for Prosperity. Nueva York: Basic Books.

Polanyi, K. (2000). A grande transformação: as origens da nossa época (9a ed.). Rio de Janeiro: Editora Campus.

Powel, W. W. (1990). Neither market nor hierarchy: network forms of organization. En B. M. Staw y L. L. Cummints (Eds.), Research in Organizational Behavior (vol. 12, pp. 295-336). Greenwich, Cт: CAI Press.

Secretaria Nacional de Economia Solidária. (2010). II Conferência Nacional de Economia Solidária. Documento Base. Brasília: SENAEs/MTE.

Sonnino, R. y Marsden, T. (August, 2005). Beyond the Divide: Rethinking Relationships between Alternative and Conventional Food Networks. Europe. Journal of Economic Geography, 6(2), 181-199.

Tygel, D. (2010). Fluxos e informações na Economia Solidária: um novo olhar sobre as práticas. Cartilha Economia Solidária, 1. Recuperado de http://pt.scribd.com/doc/51 755076/Cartilha-Economia-Solidaria-n\%C2\%BA1. 